\title{
CLOCK-induced upregulated HMGB1 resulting in circadian rhythms disruption of inflammatory microglia contributes to non-dipper hypertension in stressed rats
}

\author{
Shutian Zhang, Li Hu, Chunmei Xia
}

The authors have withdrawn their manuscript as they have discovered some potential inconsistencies in their results after further experimentation. Therefore, the authors do not wish this work to be cited as reference for the project. 